\title{
AUTOMATIZAÇÃO APLICADA NO DIMENSIONAMENTO DE UM MODELO VIRTUAL DE ÁGUAS PREDIAIS, RECORRENDO À PROGRAMAÇÃOO VISUAL
}

\author{
Ricardo Simões ${ }^{(1)}$ \\ (1) Entusiasta da automatização na conceção de projetos, Lisboa
}

\begin{abstract}
Resumo
A automatização consiste na utilização da tecnologia com vista à concretização de tarefas, por norma, realizadas manualmente. A sua implementação permitirá a otimização de procedimentos na fase de conceção de projetos, procurando assim maximizar a produtividade e promover um elevado nível de qualidade, uma vez que reduz significativamente a probabilidade de ocorrência de erros, assim como o tempo necessário à execução dessa tarefa. Sendo os modelos virtuais um dos pilares fundamentais do Building Information Modeling (BIM), o desenvolvimento de algoritmos no âmbito do dimensionamento de projetos de especialidades permite tirar partido das vantagens associadas à sua automatização e da informação associada a elementos dos respetivos modelos.

Este artigo vem apresentar uma metodologia constituída por um conjunto de algoritmos desenvolvidos com base em programação visual, recorrendo ao Dynamo, com vista à automatização do processo de dimensionamento de um modelo virtual referente à especialidade de Águas Prediais elaborado no Revit, ambos softwares comercializados pela Autodesk.
\end{abstract}

\section{Introdução}

A digitalização no setor da Arquitetura, Engenharia e Construção (AEC) é habitualmente associada a metodologias como o BIM, uma vez que têm por base a modelação de protótipos virtuais e de toda a informação a eles alusiva, mediante um conjunto de regras e normas.

Entre as inúmeras vantagens conhecidas relativamente à utilização de modelos virtuais, no âmbito da conceção de projeto, destaca-se a possibilidade de associar aos seus elementos todo o tipo de dados, permitindo assim explorar a automatização no processo de modelação dos respetivos protótipos.

A implementação da automatização reside na substituição da ação humana pela utilização da tecnologia na realização de uma determinada tarefa, através da criação de rotinas com o objetivo de garantir a execução de um conjunto de ações sequenciais, a fim de se atingir um determinado resultado. 
No âmbito da conceção de projetos, o processo de dimensionamento pode definir-se, de uma forma genérica, pela seguinte sequência de ações:

- Caracterização dos elementos e respetivos parâmetros de projeto;

- Dimensionamento; e,

- Verificação e validação de resultados.

Pelo facto de se tratar de um processo repetitivo, o desenvolvimento de um conjunto de algoritmos permite automatizar e otimizar a realização das diferentes tarefas a executar.

Em alternativa à programação tradicional, surge a programação visual. Esta consiste numa linguagem de programação que permite descrever processos através de elementos gráficos.

Desta forma, o desenvolvimento de algoritmos torna-se numa tarefa menos complexa, uma vez que recorrendo à programação visual, é possível descrever todo o processo de dimensionamento de uma forma humanamente intuitiva, desenvolvendo o algoritmo de acordo com as diferentes ações sequenciais que definem os respetivos procedimentos.

Neste artigo é apresentada a aplicação da automatização no dimensionamento de um modelo virtual no âmbito da especialidade de Águas Prediais criado no Revit, através de um conjunto de algoritmos desenvolvidos com recurso ao Dynamo, aplicando a programação visual.

\section{Sistemas de Águas Prediais}

Um sistema de águas prediais representa toda a rede de uma propriedade no seu domínio privado, desde a respetiva ligação ao ramal público. Os seus elementos são divididos em duas categorias: nós e troços.

Genericamente, um troço é o conjunto de tubagens colineares de características iguais, como o valor de caudal, diâmetro e o respetivo material. Sendo que, os nós representam os pontos de extremidade do sistema, ou seja, os nós de entrada e de saída, assim comos os intermédios, estabelecendo a ligação entre os diferentes troços. De uma forma geral, os nós podem ser equipamentos ou acessórios, como por exemplo equipamentos sanitários ou mecânicos, válvulas ou tês (Figura 1).

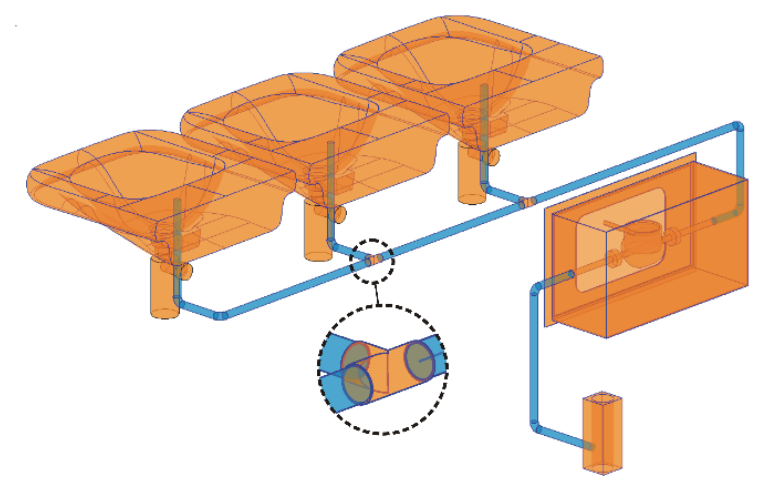

Figura 1: Elementos de um sistema de Águas Prediais: nós (laranja) e troços (azul).

No que diz respeito a legislação e normas, a conceção de sistemas de abastecimento de águas prediais deve respeitar o Regulamento Geral dos Sistemas Públicos e Prediais de Distribuição 
de Água e de Drenagem de Águas Residuais [1], assim como os regulamentos municipais aplicáveis.

\subsection{Processo de dimensionamento hidráulico}

A primeira etapa do processo de dimensionamento hidráulico consiste na caracterização de cada troço, definindo o seu valor de caudal de abastecimento e o material da tubagem. Este caudal, também denominado por caudal acumulado, corresponde à soma dos caudais instantâneos dos equipamentos a jusante do respetivo troço.

Posteriormente, o caudal acumulado é afetado por um fator de simultaneidade, parâmetro que mede a probabilidade de todos os dispositivos funcionarem ao mesmo tempo, de forma a determinar o valor do caudal de cálculo.

O dimensionamento hidráulico da secção transversal dos troços é realizado tendo como referência os valores de:

- Caudal de cálculo;

- Velocidade de escoamento; e,

- Coeficiente de rugosidade do material das tubagens.

Os cálculos seguintes definem os valores de diâmetro interno mínimo e máximo admissíveis por troço, consoante o caudal de cálculo e a velocidade de escoamento definida. Obtidas as dimensões limite da secção transversal, recorrendo a um catálogo comercial de tubagens com o mesmo material, define-se o valor do diâmetro nominal, calculando-se depois os valores das perdas de carga correspondentes.

A etapa seguinte consiste no cálculo da pressão disponível nos equipamentos, cuja validação é garantida se os valores se enquadrarem com os limites regulamentares. Não cumprindo esta verificação, deve-se proceder às devidas alterações, seja através da alteração do traçado ou com a incorporação de equipamentos, de forma a garantir o valor da pressão mínima e máxima regulamentar em todos os equipamentos.

Em suma, o processo de dimensionamento é constituído por um fluxo de trabalho definido por um conjunto de ações lógicas que dependem de regras de entrada, de processamento e de saída. Ao executar estas etapas sequencialmente, todo o processo de dimensionamento é realizado de forma algorítmica.

\section{Programação visual}

A programação visual é um tipo de programação que permite ao utilizador desenvolver um algoritmo, não sendo estritamente necessário que este possua conhecimentos de linguagem de código, uma vez que não implica o uso da programação tradicional com base em texto, mas de elementos gráficos [2].

Desta forma, a interação entre o utilizador e o programa torna-se menos complexa, dado que a utilização da programação visual permite a um software interpretar a linha de raciocínio do utilizador, através da programação associada aos elementos gráficos usados na definição do fluxo de tarefas a executar. 


\subsection{Dynamo}

O Dynamo, tal como outros softwares semelhantes, foi desenvolvido no âmbito da programação visual permitindo ao utilizador desenvolver os seus próprios algoritmos, sejam de maior ou menor complexidade, recorrendo a um conjunto de comandos de modo a definir o respetivo workflow [3], seja através do processamento de dados ou da manipulação de geometrias.

Apesar de comercializado pela Autodesk, a aplicação do Dynamo não se restringe à interação entre softwares desta empresa, como o Revit, Maya ou o Civil 3D, sendo também possível a partilha de informação com o Excel, por exemplo.

O Dynamo tem a capacidade de comunicar com diferentes softwares através das suas APIs (Application Programming Interfaces), o que permite estabelecer uma correta comunicação entre os softwares, funcionando como um plugin.

Relativamente à sua funcionalidade, é constituído por uma biblioteca de nós, denominação atribuída aos diferentes comandos, organizada por diferentes categorias consoante a sua aplicação e respetivo âmbito. Cada comando executa uma operação, normalmente em consonância com o seu nome, sendo necessário, na sua grande maioria, definir os respetivos inputs para que ele execute a respetiva função.

Através da ligação entre os diferentes nós, realizada com recurso a linhas retas ou curvas dependendo da sua configuração, é definido o workflow do algoritmo desenvolvido (Figura 2).

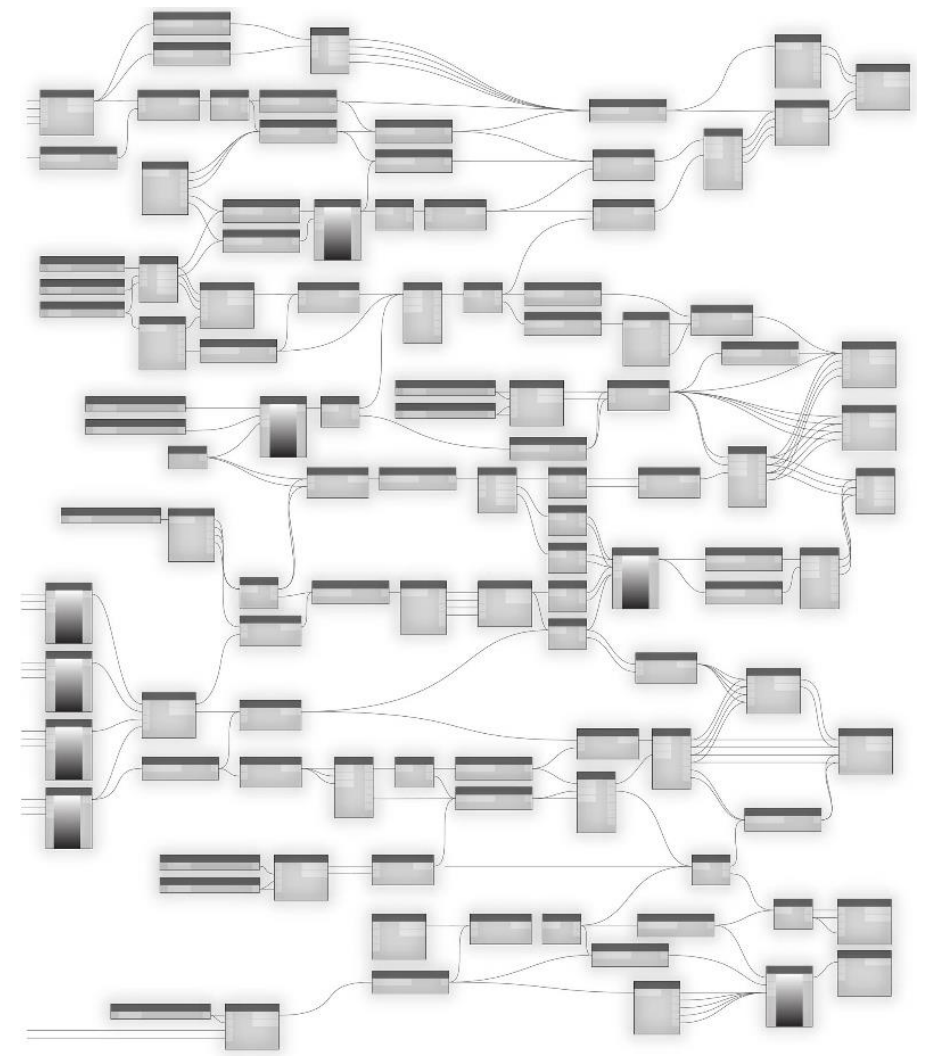

Figura 2: A anatomia da programação visual (primer.dynamobim.org, 2020).

Apesar do Dynamo possuir por defeito uma biblioteca de comandos, é possível adicionar-lhe packages. Estes são conjuntos de comandos desenvolvidos por diferentes utilizadores, encontrando-se, na sua grande maioria, disponíveis livremente na comunidade de Dynamo. 
A execução dos algoritmos, no que se refere à interação com o Revit, pode ser efetuada através do próprio Dynamo, ou recorrendo ao Dynamo Player. Este último, apresenta uma lista de rotinas que se encontram dentro de uma diretoria definida, permitindo escolher uma, definir os seus inputs, caso seja necessário, e executá-la como se de uma música ou vídeo se tratasse.

\section{Automatização no processo de dimensionamento hidráulico}

Como descrito nos capítulos anteriores, todo o processo tem, por defeito, uma estrutura algorítmica, se a sua execução depender de um workflow definido por um conjunto de ações lógicas e sequenciais obedecendo a procedimentos de entrada, de processamento e de saída. Nesse sentido, o presente artigo apresenta uma metodologia cujo objetivo é o de automatizar o processo de dimensionamento de um modelo virtual no âmbito da especialidade de Águas Prediais, utilizando algoritmos desenvolvidos através da aplicação da programação visual, recorrendo ao Dynamo.

\subsection{Metodologia}

A metodologia desenvolvida visa automatizar o processo de dimensionamento hidráulico através de um conjunto de algoritmos, com base num modelo virtual (Figura 3).

Nesse sentido, e tirando partido da potencialidade relativamente à partilha da informação entre o Revit e o Dynamo, foram desenvolvidos dois métodos de dimensionamento. O primeiro pretende, de uma forma mais célere, dimensionar os elementos do modelo utilizando apenas um algoritmo. Por outro lado, o segundo método, constituído por dois algoritmos, é mais meticuloso, uma vez que envolve a tomada de decisão por parte do projetista, recorrendo a uma folha de cálculo, sendo por isso necessário exportar a informação e, depois de efetuado o dimensionamento e respetiva validação, importar os dados para o modelo.

Atribuídas as dimensões aos elementos, nomeadamente às tubagens e aos acessórios, e independentemente do método utilizado, é calculada a pressão disponível nos equipamentos recorrendo a um outro algoritmo.

A verificação final é realizada também de modo visual, utilizando os filtros de visualização do Revit, associando a cada parâmetro de cálculo as respetivas condições de verificação relativamente aos valores regulamentares.

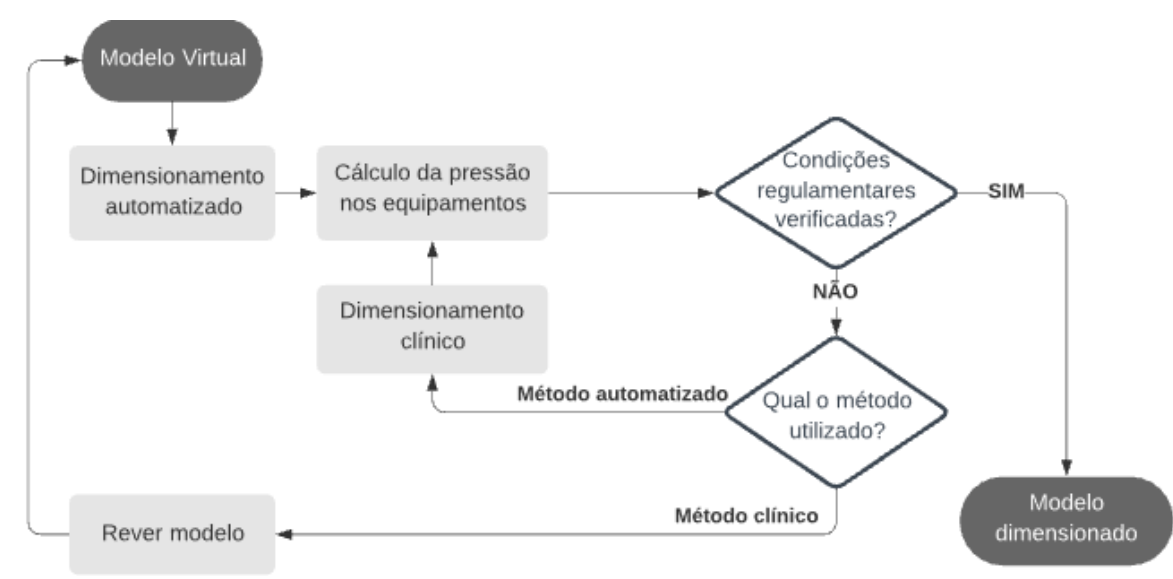

Figura 3: Fluxograma da metodologia desenvolvida. 


\subsection{Configurações do Revit no âmbito de Sistemas de Águas Prediais}

Os métodos de dimensionamento hidráulico aplicados no Revit têm, por defeito, em consideração normas e regulamentos internacionais, como o International Plumbing Code (IPC) [4]. Esta regulamentação define, por exemplo, que o caudal de um determinado equipamento é estimado com base em Fixture Units, cujo valor depende da sua tipologia, do âmbito da sua utilização (privada, pública, escritório, restauração, etc.) e ainda do tipo de elementos que controlam o seu abastecimento, possuindo por vezes diferentes ponderações, caso se trate de abastecimento de água fria ou quente [5].

Esta dependência de inúmeras variáveis para a determinação do valor de caudal de cada equipamento, apresenta-se desde logo como uma limitação na utilização das funcionalidades do Revit para efeitos de dimensionamento face ao Regulamento Geral dos Sistemas Públicos e Prediais de Distribuição de Água e de Drenagem de Águas Residuais [1], uma vez que, cada tipo de equipamento tem associado um valor mínimo, independentemente da utilização, ocupação e condições de abastecimento. $\mathrm{O}$ mesmo se verifica relativamente a outro tipo de considerações no Revit que não se enquadram com a legislação em vigor em Portugal.

No entanto, é possível definir um valor de caudal para cada elemento, também denominado por Plumbing Fixtures, através dos seus conectores. Desta forma, se todos os elementos do modelo se encontrarem corretamente conectados, as tubagens irão assumir um caudal, cujo valor corresponde à soma dos caudais dos equipamentos que se encontram a jusante. Esta funcionalidade permite obter para cada tubagem o valor do seu caudal acumulado.

Assim, apesar das configurações nativas do Revit não se enquadrarem com a legislação em vigor em Portugal para efeitos de dimensionamento de Sistemas de Águas Prediais, torna-se possível com as devidas alterações, seja nas propriedades do modelo ou nos respetivos elementos, preparar os dados necessários à execução desses cálculos, de acordo com o processo de dimensionamento descrito no subcapítulo 2.1.

\subsection{Algoritmos desenvolvidos}

No âmbito da automatização aplicada ao dimensionamento hidráulico de um modelo virtual, recorrendo à programação visual, foram desenvolvidas três rotinas principais, como referido anteriormente.

No entanto, foram também criadas outras de utilização secundária, como por exemplo para criar os parâmetros de cálculo no modelo referentes à especialidade em causa, associando-os ao respetivo tipo de elementos.

Na elaboração dos algoritmos foram utilizados alguns nós de packages como o MEPover [6] e consultados fóruns referentes ao Dynamo [7 e 8], onde a comunidade partilha conhecimento e experiências, tal como presta auxílio a eventuais dúvidas ou dificuldades que surgem no desenvolvimento de rotinas neste software.

\subsubsection{Dimensionamento Automatizado}

A fase inicial do algoritmo é constituída pela definição dos inputs, através da obtenção dos dados referente ao modelo virtual, nomeadamente a identificação dos elementos a dimensionar, importando os parâmetros essenciais à execução do algoritmo, como o caudal acumulado e material das tubagens.

Relativamente à velocidade de escoamento, um dos parâmetros de referência para efeitos de dimensionamento da secção transversal de uma tubagem, é atribuído ao utilizador a opção de definir o seu valor a considerar, restringindo-o aos limites regulamentares. 
Posteriormente, com base nos parâmetros importados, são calculados os restantes, de acordo com a legislação em vigor em Portugal, determinando para todas as tubagens o seu valor de caudal de cálculo e de seguida o seu diâmetro mínimo admissível, tendo em consideração o valor definido para a velocidade de escoamento.

Recorrendo à API do Revit são obtidas as famílias de tubagens, denominadas por Segments, e respetivos diâmetros, selecionando depois o primeiro diâmetro que cumpre o valor do diâmetro mínimo admissível calculado.

O algoritmo termina com a exportação dos valores dos parâmetros calculados, incluindo a alteração dos diâmetros das tubagens e respetivos acessórios.

A Figura 4 ilustra o algoritmo desenvolvido no Dynamo, onde se encontram definidos grupos de nós, associados a cores específicas, de acordo com a função a executar.

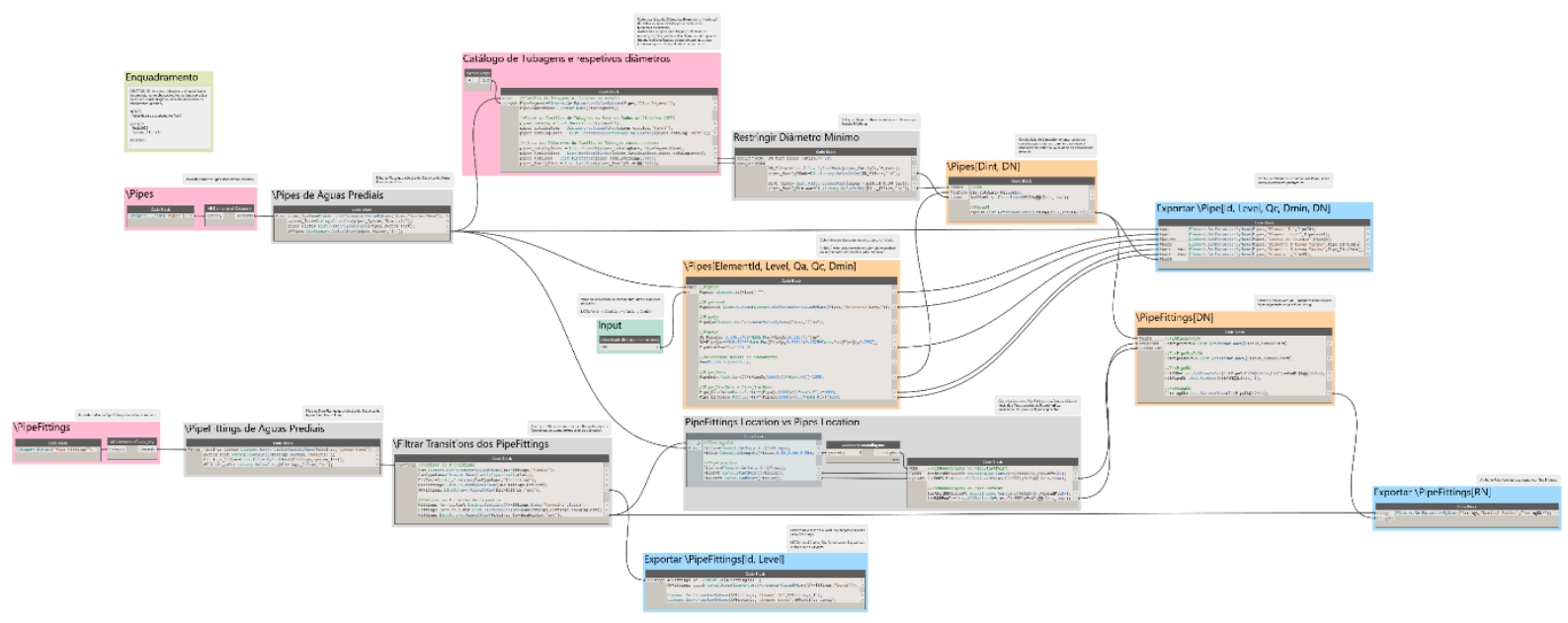

Figura 4: Workflow do Dimensionamento Automatizado.

\subsubsection{Dimensionamento Clínico}

De uma forma geral, os inputs são os considerados no algoritmo do Dimensionamento Automatizado, aos quais são ainda adicionados os equipamentos utilizados no modelo e respetivos parâmetros.

Relativamente à velocidade de escoamento, são admitidos os seus limites regulamentares [1], não sendo por isso definido um valor específico para este parâmetro.

Uma vez que este método de dimensionamento é mais minucioso, o seu workflow torna-se mais complexo, sendo necessária a elaboração de dois algoritmos. O primeiro tem como objetivo a caracterização do modelo e preparação da informação com vista à exportação dos dados para uma folha de cálculo. Informação essa que, após a devida análise e validação de resultados, será importada para o modelo no Revit, através do segundo algoritmo.

Outra grande diferença entre este método e o Dimensionamento Automatizado, é a organização das tubagens por troços, considerando o System e o Section a que pertencem, uma vez que o processo de dimensionamento hidráulico é executado por troços, ao contrário da rotina anterior que não apresenta essa necessidade.

A consideração dos limites regulamentares da velocidade de escoamento, permite determinar, de acordo com o respetivo caudal de cálculo, os valores mínimo e máximo para o diâmetro interior da secção transversal por troço. Sendo, de seguida, confrontados com os diâmetros de 
cada tipo de tubagem, importados através da API do Revit, de forma a identificar, por troço, os seus diâmetros admissíveis.

Posteriormente, para cada troço e respetivos diâmetros admissíveis, são calculados os valores de velocidade de escoamento, assim como as perdas de carga unitárias e contínuas, considerando as dimensões dos elementos no modelo.

Paralelamente, são criadas relações de dependência entre os diversos elementos, de forma a mapear o modelo, identificando a sequência de sistemas e troços desde a origem, de montante para jusante.

Desta forma, e tendo em consideração este tipo de relações, sejam geométricas ou recorrendo aos respetivos parâmetros, além de serem calculadas as cargas hidráulicas associadas a cada equipamento, tendo em consideração as suas cotas altimétricas e a pressão disponível à entrada da rede, são ainda criadas matrizes que definem analiticamente o modelo geométrico desenvolvido no Revit, resultando num algoritmo mais extenso e com um maior grau de complexidade, quando comparado com o do Dimensionamento Automatizado, tal como representa a Figura 5.

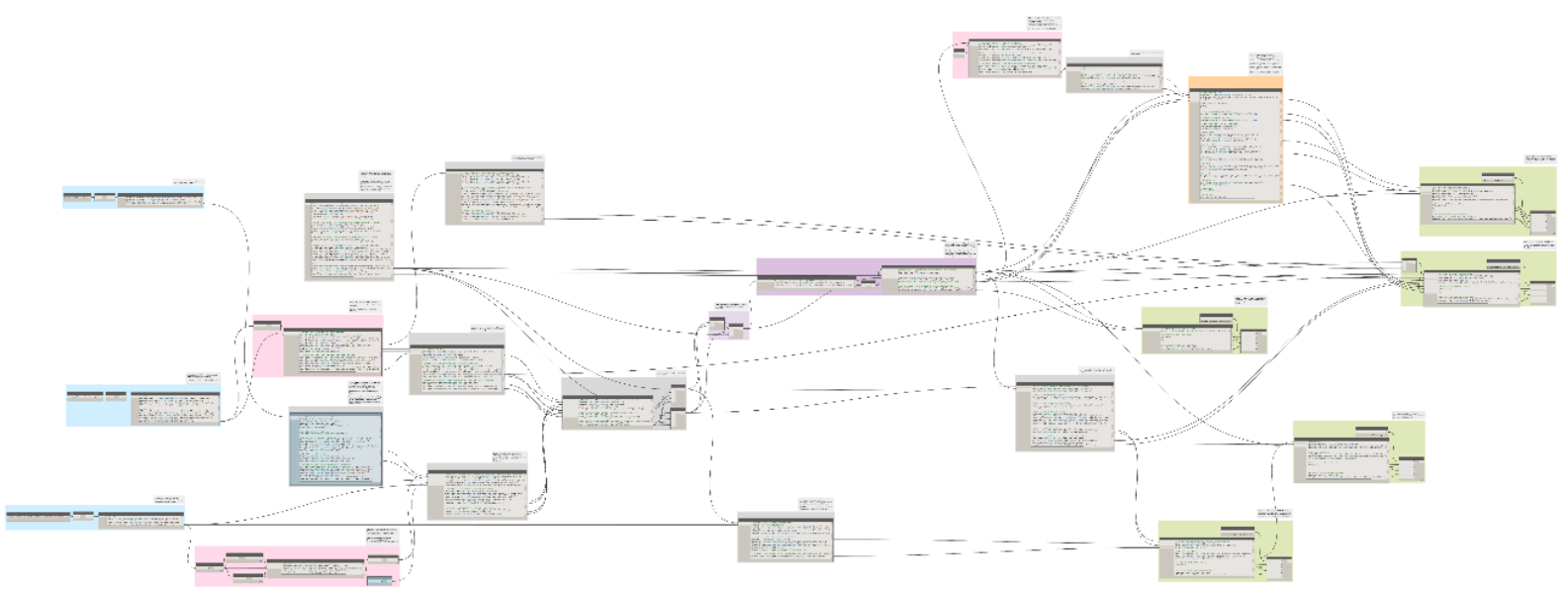

Figura 5: Workflow do primeiro algoritmo referente ao Dimensionamento Clínico.

O primeiro algoritmo termina com a exportação dos dados para uma folha de cálculo, onde será posteriormente realizado um estudo, troço a troço, dos diâmetros admissíveis e do valor da pressão disponível nos equipamentos através da perda de carga correspondente aos parâmetros considerados.

Após seleção dos diâmetros e validação dos valores de pressão em todos os equipamentos, tendo em consideração os valores regulamentares, será executado o segundo algoritmo (Figura $6)$.

Os inputs considerados para este algoritmo são os elementos a dimensionar (tubagens e acessórios), aos quais serão associados os parâmetros importados da folha de cálculo, exportando depois toda a informação para o modelo no Revit. 


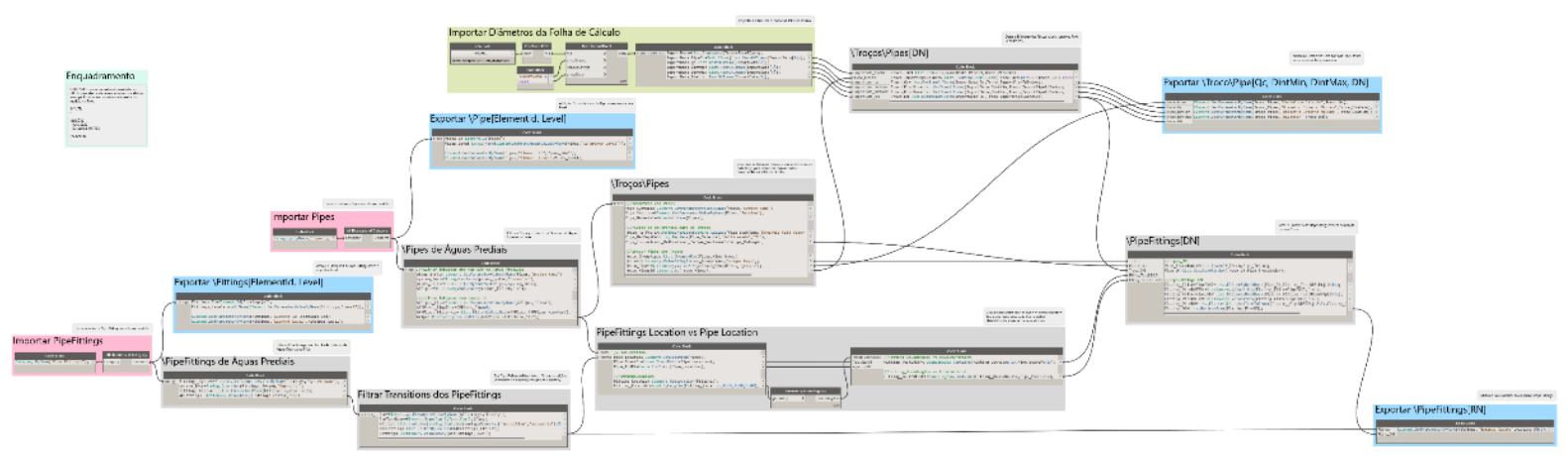

Figura 6: Workflow do segundo algoritmo referente ao Dimensionamento Clínico

\subsubsection{Cálculo da pressão nos equipamentos}

Após atribuídas as dimensões das secções transversais aos respetivos elementos no modelo, independentemente do método de dimensionamento utilizado previamente, é necessário proceder ao cálculo do valor da pressão disponível nos equipamentos, atendendo às alterações provocadas no modelo face às novas dimensões dos elementos, como por exemplo, a dimensão dos acessórios que originam uma ligeira redução no comprimento das tubagens.

No que se refere à estrutura do algoritmo (Figura 7), muito idêntica à do primeiro algoritmo do Dimensionamento Clínico, são identificados os elementos do modelo, assim como os respetivos parâmetros, procedendo depois ao cálculo da velocidade de escoamento e das perdas de carga unitárias e contínuas, referentes ao novo diâmetro de cada troço, tendo em consideração os parâmetros anteriormente determinados, como o caudal de cálculo, diâmetros interiores admissíveis e coeficiente de rugosidade do material da tubagem.

A criação das relações de dependência entre os diversos elementos, através da elaboração matrizes que permitem gerar um modelo analítico representativo do geométrico, torna possível o cálculo dos valores da pressão disponível nos diversos equipamentos, à semelhança do realizado no Dimensionamento Clínico.

No cálculo da pressão disponível nos equipamentos, poderá ser também considerada a rede de águas quentes sanitárias que, apesar de não ser um requisito para a validação do dimensionamento da secção transversal, permite um estudo complementar no que diz respeito à fase de utilização/operação do empreendimento.

Depois de realizados todos os cálculos no âmbito do dimensionamento hidráulico, assim como as verificações complementares definidas, são exportados os valores dos respetivos parâmetros para o modelo, associando-os aos elementos correspondentes.

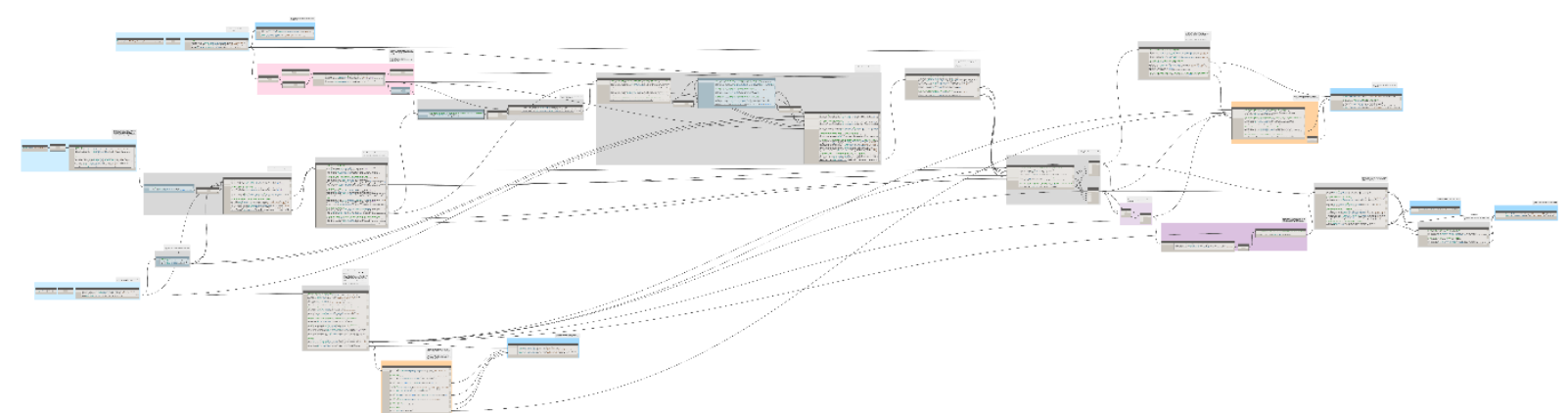

Figura 7: Workflow do cálculo da pressão nos equipamentos 


\section{Considerações finais}

A programação visual, uma vez que não obriga ao conhecimento de linguagens de código para os desenvolver, permite aos utilizadores criarem algoritmos de uma forma mais acessível e em consonância com as suas metodologias. Assim, com a sua utilização é possível automatizar determinadas tarefas que resultam da execução de etapas sequenciais definidas por um conjunto de ações lógicas de acordo com diferentes tipos de regras. Um exemplo claro deste tipo de tarefas é o processo de dimensionamento, independentemente da especialidade em causa.

Nesse sentido, foi desenvolvida uma metodologia no âmbito do dimensionamento hidráulico de um modelo virtual referente à especialidade de Águas Prediais.

A utilização dos algoritmos criados permitiu identificar vantagens relevantes do ponto de vista da gestão na sua implementação, como o aumento considerável da produtividade, uma vez que o período de duração necessário à execução das tarefas é extraordinariamente menor, e a redução da probabilidade de ocorrência de erros, o que contribui para o aumento considerável da qualidade das tarefas a realizar.

Especificamente no âmbito da especialidade de Águas Prediais, um grande benefício resultante da utilização de algoritmos está relacionado com a possibilidade de realizar verificações adicionais, como por exemplo, o cálculo da pressão disponível nos equipamentos considerando também a rede de águas quentes sanitárias.

Relativamente a potencialidades ainda por explorar, serve de exemplo, a utilização de uma base de dados de equipamentos que, em caso de necessidade e atendendo aos respetivos parâmetros, através de um algoritmo pode apresentar um conjunto de soluções que satisfaçam as necessidades mediante os valores de cálculo determinados.

Desta forma, é possível concluir que, a utilização de ferramentas com vista à automatização no âmbito da conceção de projetos, permite otimizar procedimentos e os respetivos recursos necessários à execução das respetivas tarefas e aumentar o rigor, bem como a amplitude das verificações a realizar, dinamizando todo o processo de dimensionamento.

\section{Referências}

[1] Decreto Regulamentar n. ${ }^{\circ} 23 / 1995$ de 23 de agosto

[2] Revell, Matthew, "What is visual programming?” OutSystems/blog/Dev Zone, Agosto de 2019.

[3] Dynamo Primer: https://primer.dynamobim.org/index.html

[4] Autodesk Knowledge Network: https://knowledge.autodesk.com/support/revit-products/, “About Pipe Sizing”, 8 de Maio de 2020

[5] International Code Council, ICC, "International Plumbing Code 2006”, Janeiro de 2006

[6] Dynamo Package Manager: https://dynamopackages.com/

[7] Dynamo Forum: https://forum.dynamobim.com/

[8] The Building Coder - Programming Forge, BIM and the Revit API: https://thebuildingcoder.typepad.com/ 